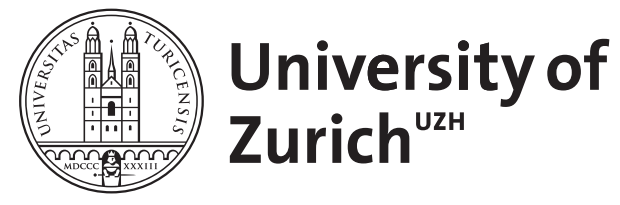

Zurich Open Repository and Archive

University of Zurich

University Library

Strickhofstrasse 39

CH-8057 Zurich

www.zora.uzh.ch

Year: 2013

Zappelphilipp beruhigen: Medikamente vs. Intervention bei den Eltern

Djalali, Sima

DOI: https://doi.org/10.1024/1661-8157/a001335

Posted at the Zurich Open Repository and Archive, University of Zurich

ZORA URL: https://doi.org/10.5167/uzh-79737

Journal Article

Accepted Version

Originally published at:

Djalali, Sima (2013). Zappelphilipp beruhigen: Medikamente vs. Intervention bei den Eltern. Praxis, 102(14):877-878.

DOI: https://doi.org/10.1024/1661-8157/a001335 


\section{Zappelphilipp beruhigen: Medikamente vs. Intervention bei den Eltern}

\section{Frage:}

Welche Evidenz gibt es für die medikamentöse Therapie von hyperaktiven Vorschulkindern gegenüber verhaltenstherapeutischen Interventionen bei den Bezugspersonen?

\section{Hintergrund:}

Immer mehr kristallisiert sich heraus, dass Aufmerksamkeitsdefizit-/Hyperaktivitäts-Syndrome (ADHS) sich im Erwachsenenalter nicht einfach «auswachsen», sondern lebenslang bestehen. Umso grösser die Bemühungen, die Störung frühzeitig zu diagnostizieren und zu behandeln. Zunehmend resultiert daraus jedoch eine Off-Label-Therapie mit Methylphenidat von Vorschulkindern mit häufigen Nebenwirkungen. Daneben existieren verschiedene Formen von verhaltenstherapeutischen Interventionen, die das soziale Umfeld des Kindes beeinflussen sollen - allen voran Elterntrainings (parent behavior training, PBT). Was bessere Ergebnisse erzielt, wurde in diesem Review mit Meta-Analyse untersucht.

\section{Einschlusskriterien:}

- Auf Englisch publizierte Studien über die Behandlung von Kindern < 6 Jahren mit bestätigter ADHS-Diagnose, ODD-Diagnose (Oppositional Defiant Disorder, "Aufsässigkeits-Trotz-Störung») oder Störungen des Sozialverhaltens gemäss DSM- oder ICD-Klassifikation oder Überweisung zum Spezialisten wegen klinisch signifikanter Hyperaktivität

- Behandlung mit Methylphenidat, Elterntraining (PBT) oder PBT in Kombination mit Interventionen Lehrpersonen und Klassenkameraden des Kindes

\section{Ausschlusskriterien:}

- Studien über alternativ- und komplementärmedizinische Behandlungen

\section{Studiendesign und Methode:}

Systematisches Literaturreview und Meta-Analyse

\section{Interventionen:}

- Systematische Literaturrecherche in den Datenbanken Medline, Embase, Cochrane, Psyclnfo und ERIC

- Standardisierte Datenextraktion und Meta-Analyse gemäss MOOSE-Guidelines

- Bewertung der Evidenz nach GRADE-Kriterien

\section{Outcome:}

Pri mär er Out come

- Beurteilung der unterschiedlichen Therapien hinsichtlich Evidenzstärke, Effektivität und Nebenwirkungen

\section{Resultat:}

- 36'888 publizierte Studienreports wurden gescreent und 55 davon in das Review eingeschlossen. 34 Berichte beschrieben reine PBT-Studien, sechs PBTKombinationsstudien und 15 Studien zu schnell freisetzenden Methylphenidatpräparaten. 13 Studien zu PBT konnten gepoolt werden für eine Meta-Analyse.

- Die Evidenzstärke für reine PBT-Studien wurde am höchsten bewertet: 32 Studien waren von hoher bis mittlerer methodischer Qualität, während zu Methylphenidat nur eine einzige methodisch akzeptable randomisierte Studie gefunden wurde sowie drei sehr kleine 
Studien (max. 44 Teilnehmer) von mittelmässiger Qualität vorlagen. Die Evidenz für PBT in Kombination mit anderen Interventionen im sozialen Umfeld war unzureichend - die Studien und Interventionsmethoden waren zu heterogen und die Ergebnisse widersprüchlich, um eine Bewertung vorzunehmen.

- Die Effektgrösse der PBT wurde anhand der Meta-Analyse von 279 Patienten bewertet und mit $-0,77(-1.21--0.34 ; 0=$ kein Effekt, $<0=$ Effekt zugunsten der PBT, $>0=$ Effekt zugunsten der Kontrollgruppe) angegeben. Für Methylphenidat konnte eine solche Berechnung mangels methodisch geeigneter Primärstudien nicht durchgeführt werden.

- Unerwünschte Nebenwirkungen wurden nur bei Methylphenidat berichtet, darunter v.a. Appetitminderung, sozialer Rückzug, Konzentrationsschwierigkeiten, Magenschmerzen/reizungen und Rebounds.

\title{
Kommentar:
}

- Sowohl PBT als auch Methylphenidat wurden in experimentellen Studien an Vorschulkindern untersucht und beide zeigten eine Dosis-Wirkungsbeziehung, die im Falle von Methylphenidat auch für Nebenwirkungen galt, während für die PBT keine unerwünschten Nebenwirkungen berichtet wurden.

- Es gab keine randomisierten Studien, die Methylphenidat und PBT direkt miteinander verglichen. Die Einzelstudien waren zu unterschiedlich in Design und Outcomes als dass ein Vergleich durch Meta-Analyse durchführbar gewesen wäre.

- Generell ist die Studienlage dünn. Zu den verhaltenstherapeutischen Interventionen gab es zwar mehr und bessere Studien als zu Methylphenidat, aber auch hier ist es heikel, generalisierende Ergebnisse abzuleiten, zu klein sind die Fallzahlen und unterschiedlich die Interventionsprogramme.

- Bei einem Fazit für die Praxis ist zu bedenken, dass verhaltenstherapeutische Interventionen aufwendig und teuer zu implementieren sind und die Abbruchrate teilweise hoch ist. Sofern entsprechende Angebote vorhanden sind, spricht die derzeitige Studienlage jedoch dafür, PBT gegenüber einer Medikation als Mittel der ersten Wahl den Vorzug zu geben. Gerade angesichts der Tatsache, dass eine reine ADHS-Diagnose bei Kindern unter sechs Jahren schwierig zu stellen ist und sich oft verschiedene soziale Entwicklungsstörungen und familiäre Probleme, die die ganze Familie betreffen, überlagern, erscheint eine a-priori-Behandlung mit Methylphenidat zu einseitig.

\section{Literatur:}

Charach A, et al.: Interventions for Preschool Children at High Risk for ADHD: A Comparative Effectiveness Review. Pediatrics 2013; Apr 1 [Epub ahead of print].

\author{
Verfasser: \\ Dr. med. Sima Djalali \\ Institut für Hausarztmedizin \\ Universität Zürich \\ Pestalozzistrasse 24 \\ 8091 Zürich \\ sima.djalali@usz.ch
}

